\title{
Kearns-Sayre's syndrome developing in a boy who survived Pearson's syndrome caused by mitochondrial DNA deletion
}

\author{
H.J. SIMONSZ, ${ }^{1}$ K. BÄRLOCHER ${ }^{2}$ \& A. RÖTIG ${ }^{3}$ \\ ${ }^{1}$ Netherlands Ophthalmic Research Institute, Amsterdam, The Netherlands; ${ }^{2}$ Ostschweizerische \\ Kinderspital, Sankt Gallen, Switzerland; ${ }^{3}$ Hopital des Enfants-Malades, Paris, France
}

Accepted 1 September 1992

Key words: Chronic progressive external ophthalmoplegia, Kearns-Sayre Syndrome, Mitochondrial DNA, Pearson syndrome

\begin{abstract}
A 7-year-old boy presented with bilateral ptosis and atypical retinitis pigmentosa. Before age two, he had had an Fe-refractory anemia, with neutropenia and thrombopenia. Just prior to the ophthalmic examination, the patient developed lactate acidosis, muscular hypotonia, ataxia and increased protein in the spinal fluid. Pancytopenia, pancreas dysfunction and growth retardation are the main features of Pearson's syndrome, most children not surviving beyond age three. The cause of Pearson's syndrome in our patient turned out to be a $5 \mathrm{~kb}$ deletion in the mitchondrial DNA. Similar deletions have been described in the Kearns-Sayre syndrome. It seems that children who survive the initial phase of Pearson's syndrome, may develop Kearns-Sayre syndrome.
\end{abstract}

\section{Introduction}

In all likelihood, the mitochondria in our cells were originally bacteria. As these bacteria entered our cells, they brought their own DNA, coding for aerobic metabolism, with them (see for short review: Palca, 1990). The 16569-base-pair (bp) long, circular, mitochondrial DNA (mtDNA) within our cells contains the code for some 13 proteins, some of which serve as (parts of) enzymes in the Krebs-cycle: NADH-dehydrogenase, succinatedehydrogenase, cytochrome-B, cytochrome-C-oxydase and ATP-synthetase. Mitochondria are inherited only maternally, via the ovum. Each cell has 2-100 mitochondria. One mitochondrion has 4-10 mtDNA copies. The mutation frequency is only approximately $1 \%$ per $10^{6}$ years but, unlike nuclear DNA, mutations in the mtDNA cannot be repaired.

In mtDNA point-mutations, as in Leber's optic neuropathy, one of the 16569 base pairs is altered. In mtDNA deletions, as in the Kearns-Sayre syndrome (progressive external ophthalmoplegia, retinitis pigmentosa, onset before age 20 , and one or more of the following: complete heart block, cerebrospinal fluid protein level above $1 \mathrm{~g} / \mathrm{L}$, and cerebellar syndrome), a piece of mtDNA is missing. The DNA with the deletion is shorter and it seems plausible that this causes it to multiply faster than the normal mtDNA 


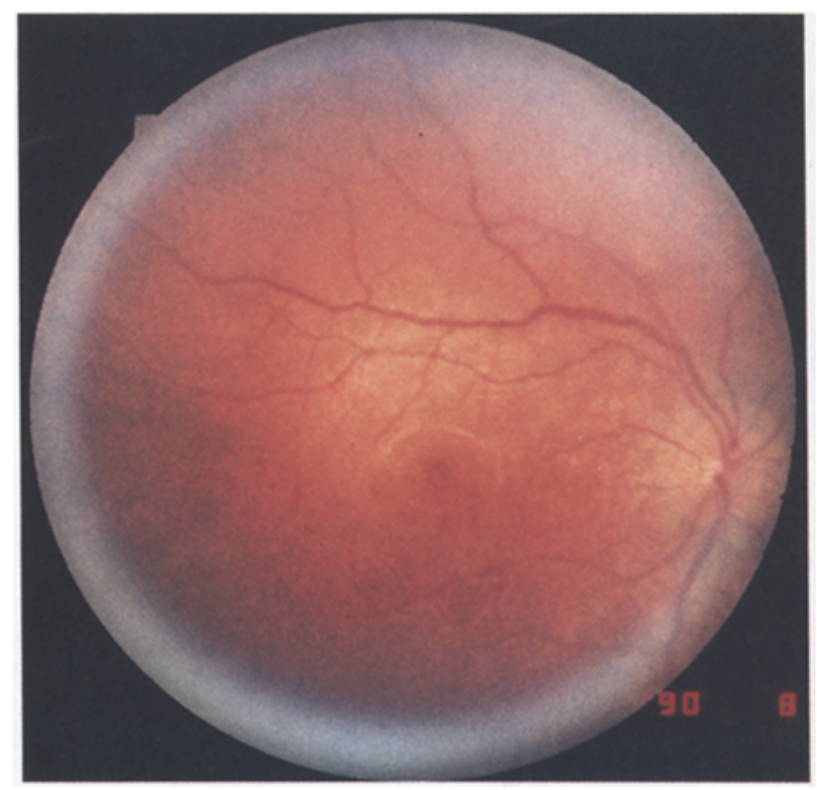

1a

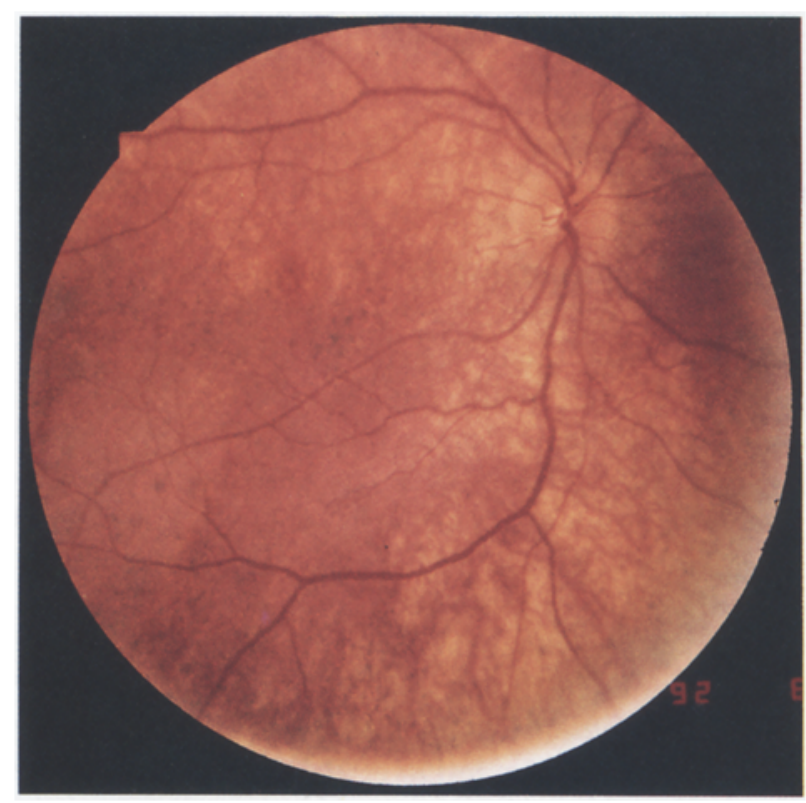

$1 b$

Fig. 1. The fundus of the right eye of the patient at presentation (panel a) showed an atypical retinitis pigmentosa, most outspoken in the periphery, but also present in the macular area. Two years later (panel b), the retinitis had progressed slightly, with more pigment clumping in the macula. The lower nasal periphery of the fundus of the left eye at presentation 


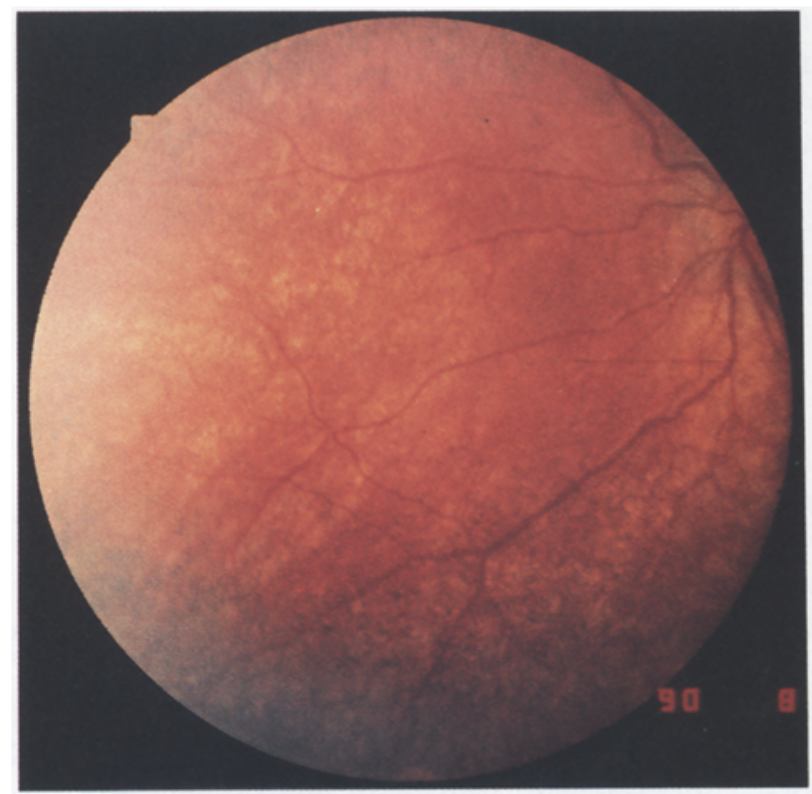

$1 \mathrm{c}$

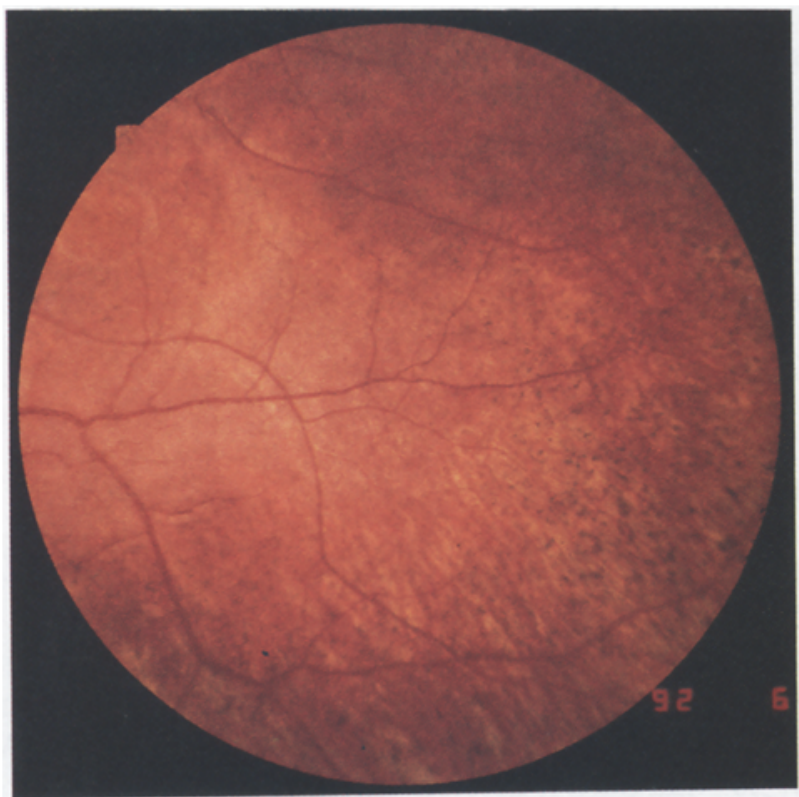

(panel c) showed the most pronounced pigment changes at the time. Two years later, the lower temporal periphery (panel d) showed similar pigment changes. As in the right eye, the macula of the left eye (upper left in panel d), now showed pigment clumping. 
in the same cell thus causing an increasing proportion of anomalous mtDNA over the years, and hence, progression of the disease. We were confronted with a case that had all the characteristics of the Kearns-Sayre syndrome but had, however, years before been diagnosed as the Pearson syndrome (Pearson et al., 1979).

\section{Case report}

A 7-year-old boy presented with bilateral incomplete ptosis and an atypical retinitis pigmentosa, more outspoken in the nasal periphery, but also affecting the central retina (Fig. 1, upper panels). Visual acuity was 1.0 in both eyes, and color vision (Ishihara plates) was normal. He had slight concentric limitation of the motility of both eyes. He had been treated previously with corticosteroid eye drops for recurring dots on the cornea associated with oedema, similar in appearance, as the referring ophthalmologist noted, to keratitis photoelectrica. Before age two, he had had anemia that could not be relieved with iron, with neutropenia and thrombopenia. The pancytopenia remitted spontaneously, but physical and mental development was retarded. At age four, the pancreas was found to be echo-dense on ultrasonography and pigment changes were noted on the skin of the arms. Just prior to the ophthalmological examination, the patient developed lactate acidosis, muscular hypotonia, ataxia and increased protein in the spinal fluid. Two years after our first examination the atypical retinitis pigmentosa had become more outspoken, with pigment clumping also occurring in the macular region (Fig. 1, lower panels). The ERG was flat: The slight concentric limitation of eye motility had hardly progressed. The ataxia and other neurologic symptoms were stationary.

\section{Methods and results}

The cause of Pearson's syndrome in our patient turned out to be a deletion in the mitchondrial DNA (described in detail by Rötig et al., 1989). Southern blot analysis of leucocyte DNA, digested with restriction enzyme PvuII, and hybridization, using a total mitochondrial probe, demonstrated a partly deleted $(11 \mathrm{~kb})$ mtDNA apart from normal mtDNA (Fig. 2). Hybridization to the partly deleted mtDNA occurred with cytochrome oxidase II and cytochrome b probes, but not with ATPase 6-cytochrome oxidase III, NADH dehydrogenase 4 and NADH dehydrogenase 5 probes. A $13 \mathrm{bp}$ direct-repeat in the wild-type mtDNA sequence was found to flank the 4977 bp deletion, that extended from bp 8483 to 13459 (Fig. 2). 


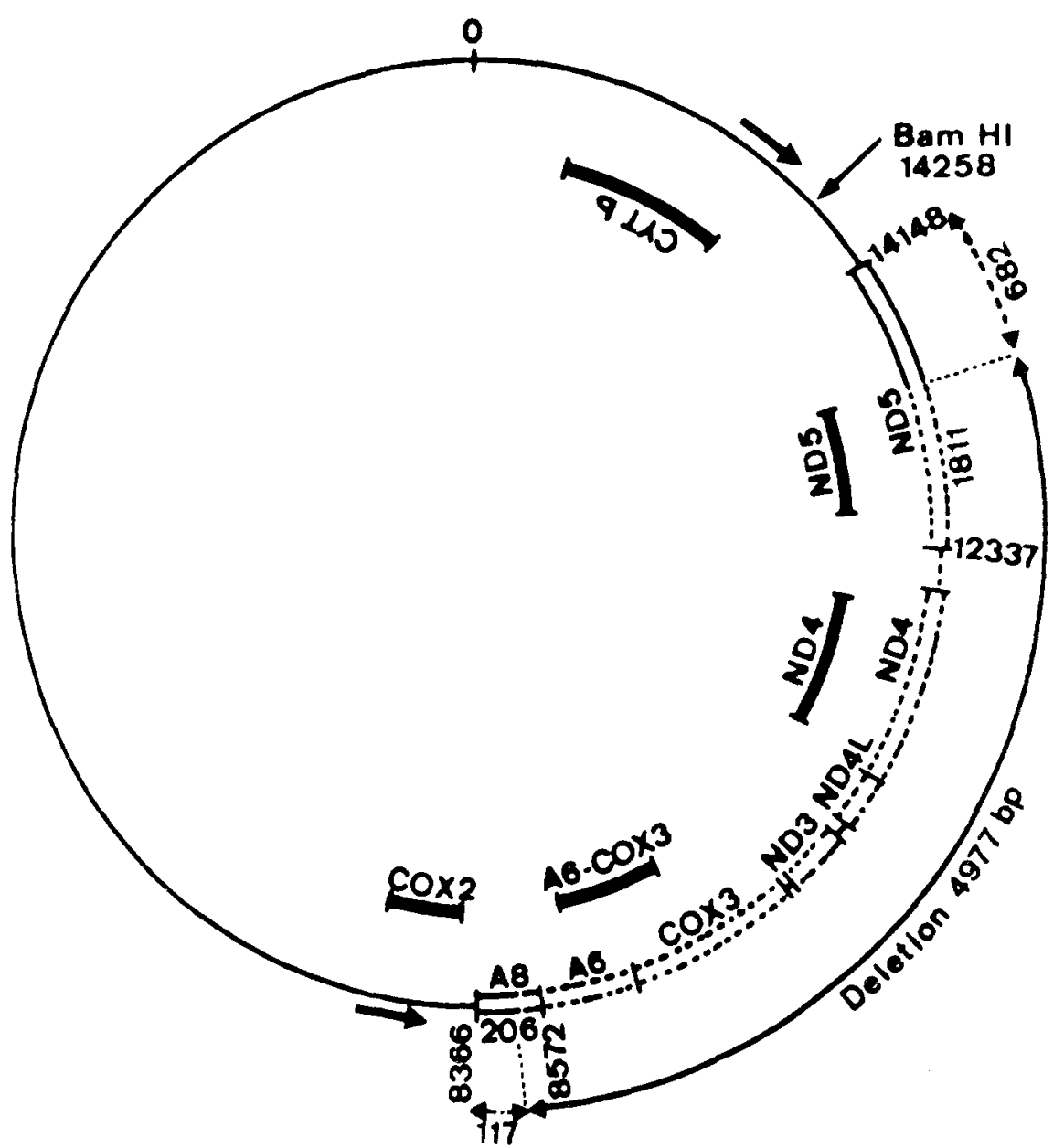

Fig. 2. The normal, circular mtDNA consisting of 16569 bp is represented in this diagram by a large circle. In the cells of the patient, apart from copies of normal mtDNA, copies of a smaller circular mtDNA were also present that backed the part of the mtDNA designated 'Deletion 4977 bp'. The parts of mtDNA coding for specific proteins are represented by a doubling of the circle and their abbreviated names (A8, A6 etcetera). The five inner bars, designated COX2, A6-COX3, etcetera represent the locations of the five probes used to determine the extent of the deletion: only cytochrome oxidase II (COX2) and cytochrome b (CYTb) probes hybridized with the mtDNA copies with the deletion; the three other probes, ATPase 6-cytochrome oxidase III (A6-COX3), NADH dehydrogenase 4 (ND4) and NADH dehydrogenase 5 (ND5), found no mirror images to bind to. (This figure was presented previously in a letter-to-theeditor describing the genetic defect of the case, Rötig et al., 1989).

\section{Discussion}

Pancytopenia, pancreas dysfunction and growth retardation are the main features of Pearson's syndrome, most children not surviving beyond age three. Recently, lactate acidosis, liver, kidney, intestinal and skin mani- 
festations have also been described. This case demonstrates that ptosis and retinitis pigmentosa, classic features of the Kearns-Sayre syndrome, can develop in children who survive the initial phase of Pearson's syndrome. The question remains to what extent these two syndromes are actually a single syndrome. In a series of studies by Moraes et al. (1989) of 123 patients with mitochondrial myopathies and encephalopathies, in 44 cases mtDNA deletions were found that varied in length between 1.3 and $7.6 \mathrm{~kb}$. All of these 44 patients had a Kearns-Sayre syndrome, probable KearnsSayre syndrome, or progressive external ophthalmoplegia. Eleven out of fourteen patients with a typical Kearns-Sayre syndrome had mtDNA deletions. The deletions were highly variable, and only one small part of the mtDNA was deleted in all cases of typical Kearns-Sayre syndrome (ND4, coding for part of NADH-dehydrogenase). Two of these eleven patients with Kearns-Sayre syndrome and a mtDNA deletion had the same mtDNA deletion as our patient. On the other hand, three out of a series of five patients with the Pearson syndrome and a mtDNA deletion (Röting, 1990), were found to have the same deletion as our patient. Why does one patient with this mtDNA deletion develops the Pearson syndrome and dies of Fe-refractory anemia at age two or three, whereas another patient develops the Kearns-Sayre syndrome? It has been suggested that heteroplasmy (different levels of anomalous mtDNA in different types of tissue like eye muscles, leucocytes or neuronal tissue) could account for the difference in phenotype. Specifically, Holt et al. (1988) found no mtDNA with deletions in leucocytes, as opposed to muscle tissue, in patients with the Kearns-Sayre syndrome. They attributed this pattern to the frequent cell division of leucocyte precursors, allowing selection against the survival of cells with deletions in mtDNA. Moraes et al., 1989, however, did find deleted mtDNA in leucocytes, but the percentage of mtDNA with deletions was much smaller than in muscle tissue.

\section{Note}

The genetic defect in this case has been described previously in a letter-to-the-editor (Rötig et al., 1989). A pediatric report of the case is in print (Baerlocher et al., 1992).

\section{References}

1. Baerlocher KE, Feldges A, Weissert HJ, Simonsz HJ, Rötig A. Mitochondrial DNA deletion in an 8-year-old boy with Pearson syndrome. J Inher Metab Dis 1992; 15 (in print)

2. Holt IJ, Harding AE, Morgan-Hughes JA. Deletions of muscle mitochondrial DNA in patients with mitochondrial myopathies. Nature 1988; 331: 717-19.

3. Moraes CT, DiMauro S, Zeviani M, et al. Mitochondiral DNA deletions in progressive 
external ophthalmoplegia and Kearns-Sayre Syndrome. New Engl J Med 1989; 320: 1293-99.

4. Palca J. The other human genome. Science 1990; 249: 1104-5.

5. Pearson HA, Lobel JS, Kocoskis SA, et al. A new syndrome of refractory sideroblastic anemia with vacuolization of marrow precursors and exocrine pancreatic dysfunction. $J$ Pediatr 1979; 95: 976-84.

6. Rötig A, Colonna M, Bonnefont JP, Blanche S, Fischer A, Sadubray JM, Munnich A. Mitochondrial DNA deletion in Pearson's marrow/pancreas syndrome (Letter). Landet 1989; 902-3.

7. Rötig A, Cormier V, Blanche S, Bonnefont JP, Ledeist F, Romero N, Schmitz J, Rustin P, Fischer A, Saudubray JM, Munnich A. Pearson's marrow-pancreas syndrome. J Clin Invest 1990; 86: 1601-8.

Address for correspondence: Dr H.J. Simonsz, Department of Ophthalmology, Academic Hospital, Pieter de Hoochweg 7-a, 3024 CN Rotterdam, The Netherlands Tel: $010-4633680 / 4766765$ 